\title{
ON THE GENERATORS OF ELEMENTARY SUBGROUPS OF GENERAL LINEAR GROUPS
}

\author{
by A. W. MASON
}

(Received 24 March, 1993)

Introduction. Let $R$ be a ring with identity and let $E_{i j} \in M_{n}(R)$ be the usual $n \times n$ matrix units, where $n \geqslant 2$ and $1 \leqslant i, j \leqslant N$. Let $E_{n}(R)$ be the subgroup of $G L_{n}(R)$ generated by all $T_{i j}(r)=I_{n}+r E_{i j}$, where $r \in R$ and $i \neq j$. For each (two-sided) $R$-ideal $\mathfrak{q}$ let $E_{n}(R, q)$ be the normal subgroup of $E_{n}(R)$ generated by $T_{i j}(q)$, where $q \in \mathfrak{q}$. The subgroup $E_{n}(R, \mathfrak{q})$ plays an important role in the theory of $G L_{n}(R)$. For example, Vaserštein has proved that, for a larger class of rings $\mathscr{C}$ (which includes all commutative rings), every subgroup $S$ of $G L_{n}(R)$, when $R \in \mathscr{C}$ and $n \geqslant 3$, contains the subgroup $E_{n}\left(R, \mathfrak{q}_{0}\right)$, where $\mathfrak{q}_{0}$ is the $R$-ideal generated by $\alpha_{i j}, r \alpha_{i i}-\alpha_{j j} r(i \neq j, r \in R)$, for all $\left(\alpha_{i j}\right) \in S$. (See [13, Theorem 1].) In addition Vaserštein has shown that, for the same class of rings, $E_{n}(R, q)$ has a simple set of generators when $n \geqslant 3$. Let $\hat{E}_{n}(R, q)$ be the subgroup of $E_{n}(R, q)$ generated by $T_{i j}(r) T_{j i}(q) T_{i j}(-r)$, where $r \in R, q \in \mathfrak{q}$. Then $\hat{E}_{n}(R, q)=$ $E_{n}(R, \mathfrak{q})$, for all $\mathfrak{q}$, when $R \in \mathscr{C}$ and $n \geqslant 3$. (See [13, Lemma 8].)

In this paper we are concerned with the question: how are $\hat{E}_{2}(R, \mathfrak{q})$ and $E_{2}(R, q)$ related? It is already known that Vaserštein's result does not in general extend to $n=2$. The author $\left[9\right.$, Example 2.6] has shown that $\hat{E}_{2}(\mathbb{Z}, \mathfrak{q})$ is of infinite index in $E_{2}(\mathbb{Z}, \mathfrak{q})$, for all but finitely many $\mathbb{Z}$-ideals $\mathfrak{q}$, where $\mathbb{Z}$ is the ring of rational integers. On the other hand Menal and Vaserštein [10, Theorem 5(a)] have proved that $\hat{E}_{2}(L, \mathfrak{q})=E_{2}(L, \mathfrak{q})$, for all $\mathfrak{q}$, where $L$ is a (possibly non-commutative) $S R_{2}$-ring. (We recall $\left[1\right.$, p. 231] that an $S R_{t}$-ring, where $t \geqslant 2$, is one which satisfies Bass's " $t$-th stable range" condition. By [1, (3.5) Theorem, p. 239] fields and semi-local rings, for example, are $S R_{2}$-rings.) Menal and Vaserštein's result however does not extend to $S R_{3}$-rings since every Dedekind ring (for example, $\mathbb{Z}$ ) is an $S R_{3}$-ring, again by [1, (3.5), Theorem, p. 239].

This paper elaborates on these results. It would appear that, unless $R$ has "sufficiently many" units, $\hat{E}_{2}(R, q)$ is likely to be of infinite index in $E_{2}(R, \mathfrak{q})$. For our first principal result, let $O(=O(d))$ be the ring of integers of $\mathbb{Q}(V-d)$, where $\mathbb{Q}$ is the set of rational numbers and $d$ is a positive integer. For each positive integer $m$, let $O_{m}$ be the order of index $m$ in $O$. (By definition, $O=O_{1}$.)

Theorem A. Suppose that $(d, m) \neq(1,1),(2,1),(3,1),(3,2),(7,1),(11,1)$. Then, for all but finitely many $\mathfrak{q}$,

$$
\hat{E}_{2}\left(O_{m}, \mathfrak{q}\right) \text { is of infinite index in } E_{2}\left(O_{m}, \mathfrak{q}\right) \text {. }
$$

Our proof is based on results of Cohn [3] and Fine [5].

For our second principal result let $D$ be a $k$-ring with a degree function, where $k$ is a field, as defined by Cohn [3, p. 21]. (The simplest examples of such rings are the polynomial rings in any number of indeterminates over $k$.)

THEOREM B. Let q be a proper D-ideal.

(i) If $\operatorname{dim}_{k}(D / \mathfrak{q})=1$, then

$$
\hat{E}_{2}(D, q)=E_{2}(D, q)
$$

Glasgow Math. J. 38 (1996) 1-10. 
(ii) Otherwise,

$$
\hat{E}_{2}(D, q) \text { is a non-normal subgroup of infinite index in } E_{2}(D, q) \text {. }
$$

Our proof is based on another result of Cohn [3].

Menal and Vaserštein's result [10, Theorem 5(a)] does extend to other rings, including some $S R_{3}$-rings, provided the rings have "many" units. For example, when $A$ is a Dedekind ring of arithmetic type with infinitely many units, it follows easily from a result of Liehl [7] that $\hat{E}_{2}(A, \mathfrak{q})=E_{2}(A, \mathfrak{q})$, for all $\mathfrak{q}$. (The simplest examples of such rings are $\mathbb{Z}[1 / p]$, where $p$ is a prime, and $k\left[t, t^{-1}\right]$ is the Laurent polynomial ring over a finite field, $k$.

We conclude by determining precisely when $\hat{E}_{2}(\mathbb{Z}, \mathfrak{q})=E_{2}(\mathbb{Z}, \mathfrak{q})$, which completes the results contained in [9, Example 2.6].

1. Orders in imaginary quadratic number fields. We begin by simplifying some of our notation.

We denote the set of units in a ring $R$ by $R^{*}$. For each $r \in R, \alpha \in R^{*}$, we put

$$
S(r)=T_{21}(r), \quad T(r)=T_{12}(r), \quad D(\alpha)=\operatorname{diag}\left(\alpha, \alpha^{-1}\right) .
$$

For each $x, y \in R$ we put

$$
S T(x, y)=S(x) T(y) S(-x) \text { and } T S(x, y)=T(x) S(y) T(-x) .
$$

Then $\hat{E}_{2}(R, \mathfrak{q})$ is generated by $S T(r, q)$ and $T S(r, q)$, where $r \in R$ and $q \in \mathfrak{q}$.

Let $d, O$ and $O_{m}$ be as above. We may assume that $d$ is square-free. Let

$$
\omega= \begin{cases}\vee-d, & d \equiv 1,2(\bmod 4), \\ (1+\sqrt{ }-d) / 2, & d \equiv 3(\bmod 4) .\end{cases}
$$

It is well-known that

$$
O_{m}=\mathbb{Z}+\omega_{m} \mathbb{Z}
$$

where $\omega_{m}=m \omega$. It follows that every non-zero $O_{m}$-ideal $\mathfrak{q}$ is a $\mathbb{Z}$-module of rank 2 and consequently is of finite index in $O_{m}$. We require a "canonical" set of $\mathbb{Z}$-generators for such a $\mathfrak{q}$.

Lemma 1.1. Let $\mathfrak{q}$ be a non-zero $O_{m}$-ideal. Then there exist unique $\alpha, \beta, \gamma \in \mathbb{Z}$ with the following properties:

(i) $\mathfrak{q}=\left(\alpha \omega_{m}+\beta\right) \mathbb{Z}+\gamma \mathbb{Z}$

(ii) $\alpha>0$ and $0 \leqslant \beta<\gamma$;

(iii) $\alpha \mid \beta$ and $\alpha \mid \gamma$;

(iv) $\left|O_{m}: \mathfrak{q}\right|=\alpha \gamma$.

Proof. From the above $\mathfrak{q}$ has $\mathbb{Z}$-generators of the form

$$
\omega_{1}=\alpha^{\prime} \omega_{m}+\beta^{\prime} \text { and } \omega_{2}=\alpha^{\prime \prime} \omega_{m}+\beta^{\prime \prime},
$$

where $\alpha^{\prime}, \beta^{\prime}, \alpha^{\prime \prime}, \beta^{\prime \prime} \in \mathbb{Z},\left(\alpha^{\prime}, \alpha^{\prime \prime}\right) \neq(0,0)$ and $\left(\beta^{\prime}, \beta^{\prime \prime}\right) \neq(0,0)$. 
We now replace $\omega_{1}, \omega_{2}$ with $\omega_{1}^{\prime}, \omega_{2}^{\prime}$ where

$$
\left[\begin{array}{l}
\omega_{1}^{\prime} \\
\omega_{2}^{\prime}
\end{array}\right]=A\left[\begin{array}{l}
\omega_{1} \\
\omega_{2}
\end{array}\right]
$$

and $A \in G L_{2}(\mathbb{Z})$. In this way we can assume $\alpha^{\prime \prime}=0, \alpha^{\prime}, \beta^{\prime \prime}>0$ and $0 \leqslant \beta^{\prime}<\beta^{\prime \prime}$. Let $\alpha^{\prime}=\alpha, \beta^{\prime \prime}=\gamma$ and $\beta^{\prime}=\beta$.

Now $\gamma \omega_{m} \in q$ and so $\alpha \mid \beta$ and $\alpha \mid \gamma$, The uniqueness of the $\alpha, \beta, \gamma$ follows, for example, from the fact that $\gamma$ is the smallest positive integer in $\mathfrak{q}$. Part (iv) is obvious.

Notation. We put

$$
q=\left(\alpha, \beta^{*}, \gamma^{*}\right),
$$

where $\beta=\alpha \beta^{*}$ and $\gamma=\alpha \gamma^{*}$. (Then $0 \leqslant \beta^{*}<\gamma^{*}$.)

The principal result of this section depends upon Cohn's theory of $G L_{2}$ over discretely normed rings. (See $[3, \S 5]$.)

To simplify our notation let

$$
U=T\left(\omega_{m}\right), \quad T=T(1), \quad A=\left[\begin{array}{rr}
0 & -1 \\
1 & 0
\end{array}\right], \quad J=-I_{2} .
$$

Lemma 1.2. Suppose $(d, m) \neq(1,1),(2,1),(3,1),(3,2),(7,1),(11,1)$.

(i) $E_{2}\left(O_{m}\right)=\left\langle U, T, A: A^{2}=(A T)^{3}=J, J^{2}=I_{2}, U T=T U, J\right.$ central $\rangle$.

(ii) $E_{2}\left(O_{m}\right)=S L_{2}(\mathbb{Z}){ }_{C} B$,

the amalgamated product of

$$
S L_{2}(\mathbb{Z})=\left\langle A ; T: A^{2}=(A T)^{3}=J, J^{2}=I_{2}, J \text { central }\right\rangle,
$$

and

$$
B=\left\langle J, U, T: U T=T U, J^{2}=I_{2}, J \text { central }\right\rangle \cong(\mathbb{Z} / 2 \mathbb{Z}) \times \mathbb{Z}^{2},
$$

over

$$
C=S L_{2}(\mathbb{Z}) \cap B=\left\langle J, T: J^{2}=I_{2}, J T=T J\right\rangle \cong(\mathbb{Z} / 2 \mathbb{Z}) \times \mathbb{Z} .
$$

Proof. Cohn [3, p. 16] has defined a discretely normed ring and Dennis [4, Theorem 3] has proved $O_{m}$ is discretely normed precisely when $(d, m)$ satisfies the above restrictions.

By virtue of [5, Theorem (5.2)] $E_{2}\left(O_{m}\right)$ has a presentation of the type described in [3, Theorem (2.2)]. Using an approach similar to that of Fine in [5, Theorem 4.8.1, p. 120] this presentation simplifies to that in (i) above.

Part (ii) follows immediately. (See also [5, Theorem 4.8 .2 (1), p. 120].)

Notation. Let $q=\left(\alpha, \beta^{*}, \gamma^{*}\right)$ as above. We put

$$
E_{2}\left(O_{m}, \mathfrak{q}\right)=E_{2}\left(\alpha, \beta^{*}, \gamma^{*}\right)
$$

It is clear that $E_{2}\left(\alpha, \beta^{*}, \gamma^{*}\right)$ is the normal subgroup of $E_{2}\left(O_{m}\right)$ generated by

$$
U^{\alpha} T^{\alpha \beta^{*}} \text { and } T^{\alpha \gamma^{*}} \text {. }
$$


A. W. MASON

LEMMA 1.3. (i) $E_{2}(\alpha, 0,1)$ is of infinite index in $E_{2}\left(O_{m}\right)$, when $\alpha \geqslant 6$.

(ii) $E_{2}\left(1, \beta^{*}, \gamma^{*}\right)$ is of infinite index in $E_{2}\left(O_{m}\right)$, when $\gamma^{*} \geqslant 6$.

Proof. (i) Let $N$ be the normal subgroup of $E_{2}\left(O_{m}\right)$ generated by $J$ and $U$ and let $G=E_{2}\left(O_{m}\right) / N$. Then, by Lemma 1.2(i),

$$
G=\left\langle a, t: a^{2}=t^{3}=1\right\rangle \cong P S L_{2}(\mathbb{Z}),
$$

where $a$ (resp. $t$ ) is the image of $A$ (resp. $T$ ) in $G$.

Now let $M$ be the image of $E_{2}(\alpha, 0,1)$ in $G$. Then $G / M$ has a presentation of the form

$$
G / M=\left\langle x, y: x^{2}=y^{2}=(x y)^{\alpha}\right\rangle,
$$

which is one of the classical triangle groups. It is a classical result that this group is infinite when $\alpha \geqslant 6$. Part (i) follows.

For part (ii) we repeat the argument with $N$ the normal subgroup of $E_{2}\left(O_{m}\right)$ generated by $J$ and $U T^{\beta^{*}}$.

Corollary 1.4. When $\alpha \geqslant 6$ or $\gamma^{*} \geqslant 6, E_{2}\left(\alpha, \beta^{*}, \gamma^{*}\right)$ is of infinite index in $E_{2}\left(O_{m}\right)$.

Proof. Follows from Lemma 1.3 since $E_{2}\left(\alpha, \beta^{*}, \gamma^{*}\right) \leqslant E_{2}(\alpha, 0,1) \cap E_{2}\left(1, \beta^{*}, \gamma^{*}\right)$.

We require one more lemma before our first principal result.

LEMma 1.5. For all ideals $\mathfrak{q}, \hat{E}_{2}\left(O_{m}, \mathfrak{q}\right)$ is finitely generated.

Proof. We may assume that $q \neq\{0\}$. Then from the above $O_{m} / \mathfrak{q}$ is finite and $q$ is a $\mathbb{Z}$-module of rank 2 . Let $\left\{a_{1}, \ldots, a_{s}\right\}$ be a set of coset representatives of $O_{m}(\bmod q)$ and let $\omega_{1}, \omega_{2}$ be a $\mathbb{Z}$-basis of $\mathfrak{q}$. Then $\hat{E}_{2}\left(O_{m}, \mathfrak{q}\right)$ is generated by

$$
S T\left(a_{i}, \omega_{j}\right) \text { and } T S\left(a_{i}, \omega_{j}\right) \text {, }
$$

where $1 \leqslant i \leqslant s$ and $j=1,2$.

THEOREM 1.6. For all but finitely many $O_{m}$-ideals $\mathfrak{q}, \hat{E}_{2}\left(O_{m}, \mathfrak{q}\right)$ is of infinite index in $E_{2}\left(O_{m}, q\right)$

Proof. We may assume that $q \neq\{0\}$. Let $q=\left(\alpha, \beta^{*}, \gamma^{*}\right)$ as above. By Lemma 1.5 it suffices to prove that $E_{2}\left(\alpha, \beta^{*}, \gamma^{*}\right)$ is infinitely generated when $\alpha \geqslant 6$ or $\gamma^{*} \geqslant 6$.

With the notation of Lemma 1.2(ii) we note that

$$
\left|C: C \cap E_{2}\left(\alpha, \beta^{*}, \gamma^{*}\right)\right|<\infty,
$$

since $T^{\alpha \gamma^{*}} \in E_{2}\left(\alpha, \beta^{*}, \gamma^{*}\right)$. Suppose that $E_{2}\left(\alpha, \beta^{*}, \gamma^{*}\right)$ is finitely generated, where $\alpha \geqslant 6$ or $\gamma^{*} \geqslant 6$. Then, combining Lemma 1.2 (ii) with a result of Karrass and Solitar $[6$, Theorem 10], we conclude that

$$
\left|E_{2}\left(O_{m}\right): E_{2}\left(\alpha, \beta^{*}, \gamma^{*}\right)\right|<\infty,
$$

which contradicts Corollary 1.4 . The result follows. 
Notes. (i) Theorem 1.6 is best possible in the sense that there are ideals $q$ for which $\hat{E}_{2}\left(O_{m}, \mathfrak{q}\right)=E_{2}\left(O_{m}, \mathfrak{q}\right)$. (Trivially, $\hat{E}_{2}\left(O_{m},\{0\}\right)=E_{2}\left(O_{m},\{0\}\right)=\left\{I_{2}\right\}$ and $\hat{E}_{2}\left(O_{m}, O_{m}\right)=$ $E_{2}\left(O_{m}, O_{m}\right)=E_{2}\left(O_{m}\right)$.)

(ii) By Lemma 1.1 it follows, for example, that Theorem 1.6 holds for all non-zero $q$ where $\left|O_{m}: \mathfrak{q}\right|>125$.

(iii) The results for $\hat{E}_{2}(\mathbb{Z}, \mathfrak{q})$ are very similar to the above and will be described in detail in the last section.

2. $k$-rings with a degree function. Throughout this section $D$ denotes a (commutative) $k$-ring with a degree function as defined by Cohn $\left[3 ;\right.$ p. 21], in which case $D^{*}=k^{*}$, where $k$ is a field. Examples of such $D$ include

(i) polynomial rings in any number of indeterminates over $k$,

(ii) the coordinate ring $C=C(\mathscr{C}, P, k)$ of the affine curve obtained by removing a closed point $P$ from a projective curve $\mathscr{C}$ over $k$. (The simplest example of type (ii) is the polynomial ring $k[t]$.)

We begin with a "positive" result.

TheOREM 2.1. Let $\mathfrak{q}$ be a $D$-ideal such that $\operatorname{dim}_{k}(D / \mathfrak{q}) \leqslant 1$. Then

$$
\hat{E}_{2}(D, \mathfrak{q})=E_{2}(D, \mathfrak{q})=E_{2}(D) \cap S L_{2}(D, \mathfrak{q})
$$

Proof. Since $\hat{E}_{2}(D, D)=E_{2}(D, D)=E_{2}(D)$ we may assume that $\operatorname{dim}_{k}(D / \mathfrak{q})=1$, i.e. $D / \mathfrak{a} \cong k$.

Let $X \in E_{2}(D) \cap S L_{2}(D, \mathfrak{q})$. Then

$$
X=T\left(x_{1}\right) S\left(y_{1}\right) \ldots T\left(x_{n}\right) S\left(y_{n}\right),
$$

where $x_{1}, y_{1}, \ldots, x_{n}, y_{n} \in D$. Now

$$
x_{i}=q_{i}+\alpha_{i} \text { and } y_{i}=\hat{q}_{i}+\beta_{i},
$$

for some $q_{i}, \hat{q}_{i} \in \mathfrak{q}$ and $\alpha_{i}, \beta_{i} \in k$, where $1 \leqslant i \leqslant n$. It is clear that $X$ can be written in the form $X=X_{1} X_{2}$, where (i) $X_{1}$ is a product of matrices of the type $Y T(q) Y^{-1}$, with $Y \in S L_{2}(k)$ and $q \in q$, and (ii) $X_{2} \in S L_{2}(k)$. (Note that $A T(x) A^{-1}=S(-x)$.) Clearly $X_{1} \in S L_{2}(D, \mathfrak{q})$. Hence $X_{2} \in S L_{2}(D, \mathfrak{q})$ and so $X_{2}=I_{2}$. It suffices therefore to prove that

$$
Y T(q) Y^{-1} \in \hat{E}_{2}(D, q) \text {. }
$$

Let

$$
Y=\left[\begin{array}{ll}
\alpha & \beta \\
\gamma & \delta
\end{array}\right]
$$

There are two possibilities.

(a) $\alpha \neq 0$ : In this case

$$
Y=S\left(\alpha^{*}\right) D(\alpha) T\left(\beta^{*}\right),
$$

where $\alpha^{*}=\gamma \alpha^{-1}$ and $\beta^{*}=\beta \alpha^{-1}$, in which case

$$
Y T(q) Y^{-1}=S T\left(\alpha^{*}, \alpha^{2} q\right)
$$


(b) $\alpha=0$ : In this case

$$
Y T(q) Y^{-1}=S\left(-\gamma^{2} q\right)
$$

The result follows.

For the simplest case, namely $D=k[t]$, Theorem 2.1 says that

$$
\hat{E}_{2}(k[t], \mathfrak{q})=E_{2}(k[t], \mathfrak{q})
$$

where $q=R$ or $(t-\alpha) R$, for some $\alpha \in k(R=k[t])$.

The situation when $\operatorname{dim}_{k}(D / \mathfrak{q})>1$ is completely different. We require another result [3] of Cohn.

Definition. Let $R$ be a ring. For each $r \in R$ we put

$$
E(r)=\left[\begin{array}{cc}
r & 1 \\
-1 & 0
\end{array}\right]
$$

Let $r, s \in R$ and $\alpha \in R^{*}$. The following identities are easily verified

$$
\begin{aligned}
E(r) E(0) E(s) & =-E(r+s), \\
E(r) D(\alpha) & =D\left(\alpha^{-1}\right) E\left(r \alpha^{2}\right) \\
E(r) E\left(\alpha^{-1}\right) E(s) & =E(r-\alpha) D\left(\alpha^{-1}\right) E(s-\alpha) .
\end{aligned}
$$

Now each element $X$ of $E_{2}(D)$ is by definition a product of matrices of the type $S(r)$ and $T(S)$ and, since $S(r)=-E(0) E(r)$ and $T(s)=-E(-s) E(0), X$ is then a product of matrices of the type $E(r)$. If such an $E(r)$, where $r \in D^{*} \cup\{0\}$, occurs in this product the above identities can be used to reduce its "length". Cohn's result says that after all such eliminations we are left with a unique standard form for $X$. form

Lemma 2.2. Let $X \in E_{2}(D)$. Then $X$ can be written uniquely in the following standard

$$
X=D(\alpha) E\left(a_{1}\right) \ldots E\left(a_{n}\right),
$$

where $\alpha \in k^{*}$ and $a_{1}, \ldots, a_{n} \in D$ such that

(i) $a_{i} \notin k$, where $1<i<n$, when $n>2$ or

(ii) $\left(a_{1}, a_{2}\right) \neq(0,0)$, when $n=2$.

Proof. See [3, Theorem (7.1)].

THEOREM 2.3. Let $\mathfrak{q}$ be a non-zero $D$ ideal, where $\operatorname{dim}_{k}(D / \mathfrak{q})>1$. Then $\hat{E}_{2}(D, \mathfrak{q})$ is a non-normal subgroup of infinite index in $E_{2}(D, \mathfrak{q})$.

Proof. Choose $x \in D$, where $x \notin \mathfrak{q} \oplus k$ and let $Y=S(x) T(x)$. Let $q \in q$, with $q \neq 0$. It suffices to prove that for each positive integer $n$

$$
Y^{n} S(q) Y^{-n} \notin \hat{E}_{2}(D, q)
$$


By means of the above identities it is clear that the standard form (Lemma 2.2) of $Y_{n} S(q) Y^{-n}$ is

$$
D(-1) E(0) E(x) E(-x) \ldots E(x) E(-x) E(q) E(x) E(-x) \ldots E(x) E(-x)
$$

(There are $2 n+2$ terms in this product.)

Suppose that $Z=Y^{n} S(q) Y^{-n}$ belongs to $\hat{E}_{2}(D, q)$. Then

$$
Z=Y_{1} \ldots Y_{S}
$$

where $Y_{i}=S T\left(a_{i}, q_{i}\right)$ or $T S\left(a_{i}, q_{i}\right)$ for some $a_{i} \notin \mathfrak{q}$ and $q_{i} \in \mathfrak{q}(1 \leqslant i \leqslant s)$, where $s \geqslant 1$. It is clear that $s>1$. We note that

$$
S T\left(a_{i}, q_{i}\right)=E(0) E\left(a_{i}\right) E\left(-q_{i}\right)\left(E\left(-a_{i}\right)\right.
$$

and

$$
T S\left(a_{i}, q_{i}\right)=E\left(-a_{i}\right)\left(E\left(q_{i}\right) E\left(a_{i}\right) E(0)\right.
$$

From the above identities it follows that

$$
X\left(r, s_{1}\right) X\left(r, s_{2}\right)=X\left(r, s_{1}+s_{2}\right),
$$

where $X=S T$ or TS. We may therefore assume that if $Y_{i}=X\left(a_{i}, \tau_{i}\right)$ and $Y_{i+1}=$ $X\left(a_{i+1}, q_{i+1}\right)$, where $X=S T$ or $T S$, and $1 \leqslant i<s$, then $a_{i} \neq a_{i+1}$. In addition, from the standard form of $Z$, we conclude that at least one $a_{i} \notin q \cup k$ (otherwise $Z \in E_{2}(q \oplus k)$ ). Assume from now on that $j$ is the largest integer with this property.

We now write $Z$ as a product of matrices of the type $E(x)$. Then

$$
Z= \pm E(a) \ldots E\left( \pm a_{j}\right) E\left(\mp q_{j}\right) E\left(\mp a_{j}\right) Y_{0},
$$

where $Y_{0} \in E_{2}(q \oplus k)$ and $Y_{0}$ or $-E(0) Y_{0} \in S L_{2}(D, q)$. Reducing this to standard form and comparing its last terms with those of (1) we conclude by Lemma 2.2 that $Y_{0}=E\left(q_{0}\right)$ or $-E(0) E\left(q_{0}\right)$, for some $q_{0} \in \mathfrak{q}$ and hence that

$$
E(x) E(-x)=E\left(\mp q_{j}+\lambda\right) E\left(\mp a_{j}+q_{0}\right),
$$

for some $\lambda \in k$. Again by Lemma 2.2 it follows that $x=\mp q_{j}+\lambda$ which contradicts the fact that $x \notin \mathfrak{q} \oplus k$.

3. Dedekind rings of arithmetic type. Throughout this section $A$ denotes a Dedekind ring of arithmetic type [2, p. 83]. By a classical theorem of Dirichlet it is known that $A^{*}$ is finite if and only if $A=\mathbb{Z}, A=O=O(d)$, for some $d$, or $A=C(\mathscr{C}, P, k)$, for some finite $k$. The preceding results (together with these of the last section) show that for most $A$ of this type the subgroup $\hat{E}_{2}(A, \mathfrak{q})$ is nearly always of infinite index in $E_{2}(A, \mathfrak{q})$.

When $A^{*}$ is infinite however the situation is completely different.

THEOREM 3.1. Let $A$ be as above and suppose that $A^{*}$ is infinite. Then, for all A-ideals $\mathfrak{q}, \hat{E}_{2}(A, \mathfrak{q})=E_{2}(A, \mathfrak{q})$.

Proof. Let $X \in E_{2}(A, q)$. Liehl [7, (20), p. 164] has proved that

$$
X=S\left(a_{1}\right) T\left(q_{1}\right) \ldots S\left(a_{n}\right) T\left(q_{n}\right)
$$


for some $a_{1}, \ldots, a_{n} \in A$ and $q_{1}, \ldots, q_{n} \in \mathfrak{q}$. Then

$$
X=S T\left(a_{1}^{*}, q_{1}\right) \ldots S T\left(a_{n}^{*}, q_{n}\right) S\left(a_{n}^{*}\right)
$$

where

$$
a_{i}^{*}=a_{1}+\ldots+a_{i} \quad(1 \leqslant i \leqslant n) .
$$

Now $X \equiv I_{2}(\bmod \mathfrak{q})$ and so $a_{n}^{*} \in \mathfrak{q}$. Hence $X \in \hat{E}_{2}(A, \mathfrak{q})$.

4. The modular group. We conclude by determining precisely when $\hat{E}_{2}(\mathbb{Z}, \mathfrak{q})=$ $E_{2}(\mathbb{Z}, \mathfrak{q})$. This completes the results contained in [9, Example 2.6]. Now $\mathfrak{q}=m \mathbb{Z}$, for some $m \geqslant 0$. We may assume that $m>0$.

LEMMA 4.1. $\hat{E}_{2}(\mathbb{Z}, m \mathbb{Z})$ is (finitely) generated by $\operatorname{ST}(a, m)$ and $T S(a, m)$, where $0 \leqslant a \leqslant m-1$

Proof. Obvious.

Lemma 4.2. (i) When $3 \leqslant m \leqslant 5, E_{2}(\mathbb{Z}, m \mathbb{Z})$ is a free group of rank

$$
1+\frac{\mu}{12}
$$

where $\mu=\left|S L_{2}(\mathbb{Z}): S L(\mathbb{Z}, m \mathbb{Z})\right|$.

(ii) When $m \geqslant 6, E_{2}(\mathbb{Z}, m \mathbb{Z})$ is a free group of infinite rank.

Proof. We denote the embedding of a subgroup $S$ of $S L_{2}(\mathbb{Z})$ in $P S L_{2}(\mathbb{Z})$ by $P S$. Now, for all $m \geqslant 3$,

$$
E_{2}(\mathbb{Z}, m \mathbb{Z}) \cong P E_{2}(\mathbb{Z}, m \mathbb{Z}),
$$

and, by [11, Theorem VIII.6, p. 143], $P E_{2}(\mathbb{Z}, m \mathbb{Z})$ is a free group.

When $1 \leqslant m \leqslant 5$ it is well-known that

$$
P E_{2}(Z, m Z)=P S L_{2}(\mathbb{Z}, m \mathbb{Z}) .
$$

(See, for example, [12, Theorem (i)].) It is known [11, Theorem VIII.7, p. 144] that the rank of $E_{2}(\mathbb{Z}, m \mathbb{Z})$ is

$$
1+\frac{\rho}{6}
$$

where $\rho=\mid P S L_{2}(\mathbb{Z}): P S L_{2}(\mathbb{Z}, m \mathbb{Z}) \|$ with $1 \leqslant m \leqslant 5$. Part (i) follows.

For part (ii) it is well-known that, when $m \geqslant 6, P E_{2}(\mathbb{Z}, m \mathbb{Z})$ is of infinite index in $P S L_{2}(\mathbb{Z})$. (See, for example, [12, Theorem (ii)].) Now $P S L_{2}(\mathbb{Z})$ is a (non-trivial) free product. (See, for example, [11, Theorem VIII.1, p. 139].) Part (ii) follows from [6, Theorem 10].

We now come to our final result.

THEOREM 4.3. (i) $\hat{E}_{2}(\mathbb{Z}, m \mathbb{Z})=E_{2}(\mathbb{Z}, m \mathbb{Z})$, when $1 \leqslant m \leqslant 4$.

(ii) When $m \geqslant 5, \hat{E}_{2}(\mathbb{Z}, m \mathbb{Z})$ is a non-normal subgroup of infinite index in $E_{2}(\mathbb{Z}, m \mathbb{Z})$.

Proof. Suppose first that $m \geqslant 6$. By Lemmas 4.1 and $4.2(\mathrm{ii}) \hat{E}_{2}(\mathbb{Z}, m \mathbb{Z})$ is a finitely 
generated subgroup of $E_{2}(\mathbb{Z}, m \mathbb{Z})$, a free group of infinite rank. Hence $\hat{E}_{2}(\mathbb{Z}, m \mathbb{Z})$ is of infinite index in $E_{2}(\mathbb{Z}, m \mathbb{Z})$ and is consequently non-normal in $E_{2}(\mathbb{Z}, m \mathbb{Z})$ by [8, Proposition 3.11, p. 17].

By Lemma 4.2(i) and [11, Theorem VII.15, p. 115] $E_{2}(\mathbb{Z}, 5 \mathbb{Z})$ is free of rank 11. By Lemma $4.1, \hat{E}_{2}(\mathbb{Z}, 5 \mathbb{Z})$ is free of rank $r$, where $r \leqslant 10$. Hence $\hat{E}_{2}(\mathbb{Z}, 5 \mathbb{Z})$ is of infinite index in $E_{2}(\mathbb{Z}, 5 \mathbb{Z})$ by $[8$, Proposition 3.9 , p. 16]. Again by [8, Proposition 3.11, p. 17] and Lemma $4.1, \hat{E}_{2}(\mathbb{Z}, 5 \mathbb{Z})$ is non-normal in $E_{2}(\mathbb{Z}, 5 \mathbb{Z})$. Part (ii) follows.

For part (i) we treat the cases $m=2,3,4$, separately. (The case $m=1$ is trivial.)

The case $m=2: E_{2}(\mathbb{Z}, 2 \mathbb{Z})$ is generated by $-I_{2}, S(2)$ and $T(2)$. (See, for example, $[12$, p. 149].) Now

$$
-I_{2}=T(2) S(-2)(T S(1,2))^{-1}
$$

and so

$$
\hat{E}_{2}(\mathbb{Z}, 2 \mathbb{Z})=E_{2}(\mathbb{Z}, 2 \mathbb{Z}) \text {. }
$$

The case $m=3$ : It is known $\left[12\right.$, p. 149] that $E_{2}(\mathbb{Z}, 3 \mathbb{Z})$ is generated by $T(3)$, $P^{-1} T(3) P, P^{-2} T(3) P^{2}$, where

$$
P=\left[\begin{array}{rr}
0 & -1 \\
1 & 1
\end{array}\right]
$$

Now $P^{-1} T(3) P=T S(-1,-3)$ and $P^{-2} T(3) P^{2}=S T(-1,-3)$ and so

$$
\hat{E}_{2}(\mathbb{Z}, 3 \mathbb{Z})=E_{2}(\mathbb{Z}, 3 \mathbb{Z})
$$

The case $m=4$ : By [11, Exercises and problems, p. 137] a complete set of right coset representatives for $S L_{2}(\mathbb{Z}, 2 \mathbb{Z})\left(\operatorname{modulo} S L_{2}(\mathbb{Z}, 4 \mathbb{Z})\right.$ ) is

$$
\pm I_{2}, \quad \pm T(2), \quad \pm S(2) \text { and } \pm T(2) S(2) \text {. }
$$

From the above $S L_{2}(\mathbb{Z}, 2 \mathbb{Z})=E_{2}(\mathbb{Z}, 2 \mathbb{Z})$ is generated by $-I_{2}, S(2)$ and $T(2)$ and so by a Reidemeister-Schreier type argument $S L_{2}(\mathbb{Z}, 4 \mathbb{Z})=E_{2}(\mathbb{Z}, 4 \mathbb{Z})$ is generated by

$$
S(4), \quad T S(2,4), \quad T(4), \quad S(2) T(2) S(-2) T(-2) \text { and } T(2) S(2) T(2) S(-2) \text {. }
$$

From the above $[T S(1,2)]^{2}=T(2) S(-2) T(2) S(-2)$ and so

$$
T(2) S(2) T(2) S(-2)=T S(2,4)[T S(1,2)]^{2} \text {. }
$$

In addition

$$
S(2) T(2) S(-2) T(-2) T S(2,4)[T S(1,2)]^{2}=S T(2,4)
$$

It follows that

$$
\hat{E}_{2}(\mathbb{Z}, 4 Z)=E_{2}(Z, 4 Z)
$$

\section{REFERENCES}

1. H. Bass, Algebraic K-theory (Benjamin, 1968).

2. H. Bass, J. Milnor and J-P. Serre, Solution of the congruence subgroup problem for $S L_{n}$ $(n \geqslant 3)$ and $S p_{2 n}(n \geqslant 2)$, Publ. Math. Inst. Hautes Étud. Sci. 33 (1967), 59-137. 
3. P. M. Cohn, On the structure of the $G L_{2}$ of a ring, Publ. Math. Inst. Hautes Étud. Sci. 30 (1966), 5-53.

4. R. K. Dennis, The $G E_{2}$-property of discrete subrings of $C$, Proc. Amer. Math. Soc. 50 (1975), 77-82.

5. B. Fine, Algebraic Theory of the Bianchi groups (Marcel Dekker, 1989).

6. A. Karrass and D. Solitar, The subgroups of a free product of two groups with an amalgamated subgroup, Trans. Amer. Math. Soc. 150 (1970), 227-255.

7. B. Liehl, On the groups $S L_{2}$ over orders of arithmetic type, J. Reine Angew. Math. 323 (1981), 153-171.

8. R. C. Lyndon and P. E. Schupp, Combinatorial Group Theory (Springer-Verlag, 1977).

9. A. W. Mason, Congruence hulls in $S L_{n}$, J. Pure Appl. Algebra. 89 (1993) 255-272.

10. P. Menal and L. N. Vaserštein, On the structure of $G L_{2}$ over stable range one rings, J. Pure Appl. Algebra 64 (1990), 149-162.

11. M. Newman, Integral Matrices (Academic Press, 1972).

12. R. A. Rankin, Subgroups of the modular group generated by parabolic elements of constant amplitude, Acta Arith. 18 (1971), 145-151.

13. L. N. Vaserštein, On the normal subgroups of $G L_{n}$ over a ring, Lecture Notes in Mathematics 854 (Springer-Verlag, 1981), 456-465.

14. K. Wohlfahrt, An extension of F. Klein's level concept, Illinois J. Math. 8 (1964), 529-535.

Department of Mathematics

UNIVERSITY OF GLASGOW

Glasgow G12 8QW

SCOTLAND

e-mail: awm@MATHS.GLA.AC.UK. 